\title{
AGE DETERMINATION AND PALAEOGEOGRAPHIC RECONSTRUCTION OF DIAPONDIA ISLANDS IN NW GREECE, BASED ON CALCAREOUS NANNOFOSSILS
}

\author{
Makrodimitras G. ${ }^{1}$, Stoykova K. ${ }^{2}$, Vakalas I. ${ }^{1}$, and Zelilidis A. ${ }^{1}$ \\ ${ }^{1}$ Laboratory of Sedimentology, Department of Geology, University of Patras, 26500, Rio, Patras, Greece, \\ ivakalas@upatras.gr,gmakrodimitr@upatras.gr,a.zelilidis@upatras.gr \\ ${ }^{2}$ Department of Paleontology, Stratigraphy and Sedimentology, Geological Institute of Bulgarian, \\ Academy of Sciences, stoykova@geology.bas.bg
}

\begin{abstract}
Diapondia islands - Ereikoussa, Othonoi and Mathraki - extended in the north-western area of Greece, are part of the external Ionian zone of the Hellenides. The exposed sedimentary succession of the three islands consists of up to $74 m$ thick submarine fans, interpreted as inner fan deposits. The determination of the sediment ages is based on the study of calcareous nannofossils, which came from almost 63 samples covering geological cross sections across the coast line of the three islands. The recorded nannofossil associations are assigned to the biozones of Martini's (1971) standard scheme. According to the age determination clastic sedimentation in the studied area started during the Late Oligocene and finished during the Early Miocene time. Moreover, the age determination and in accordance with the previous results, indicate that also the Ionian thrust activity started during Late Oligocene.
\end{abstract}

Key words: Diapondia islands, Erikoussa, Othonoi, Mathraki, calcareous nannofossils, turbidites, external Ionian zone, Ionian thrust.

\section{Introduction}

The Ionian zone has been studied in detail during the last century and is subdivided by internal thrusting into three sub-basins (internal, middle and external) (Aubouin, 1959; I.G.S.R. \& I.F.P, 1966). The studied area (North-western Greece) is a part of the external Ionian zone. Clastic sedimentation in the Ionian zone took place from middle Eocene to late Miocene in a foreland basin, the Pindos foreland (Avramidis et al., 2002; Vakalas et al., 2001) which was subdivided due to internal thrusting and changed to a complex type foreland basin. It belongs to the external Ionian zone and thus to the western part of the Pindos foreland, bounded eastwards by the middle Ionian internal thrust and westwards by the Ionian thrust. The presence of strike-slip faults, with ENE-WSW direction, internal to the foreland basin, influenced the depositional conditions in the studied area. Internal thrust activity together with the sedimentation, has influenced transportation and grain-size distribution, whereas strike-slip faults many times acting as pathways to deliver coarse-grained sediments in more distal parts (Avramidis \& Zelilidis, 2002; Vakalas et al., 2001). Many times the continuous uplift due to internal thrusting produced large slumps horizons of the uncondensed sediments, deposited over the uplifted area (Avramidis et al., 2002). 


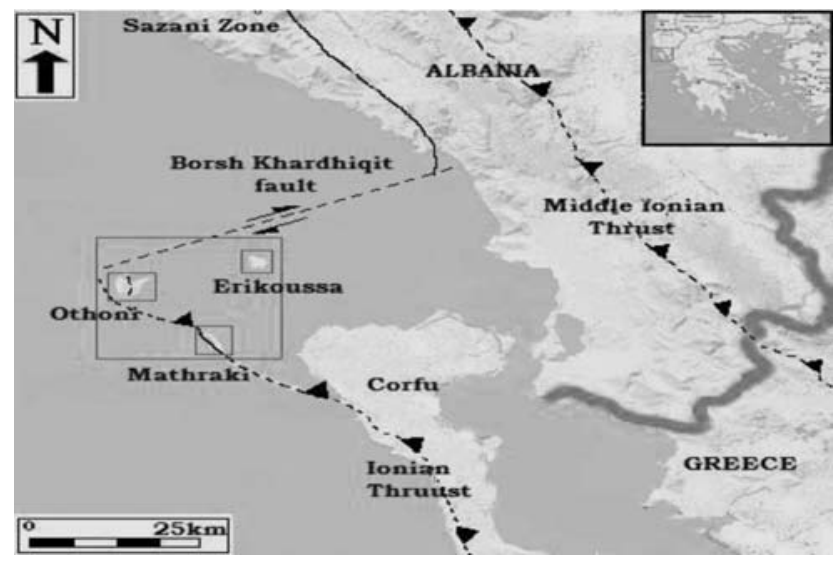

Fig. 1: Map of the studied area, where the major structural elements are presented.

The aim of this work is to determine the depositional age of the submarine fans, accumulated in the studied area. This age is based on the study of calcareous nannofossil content of the sediments. The nannofossil associations from each sample are revealed by paleontological determination of characteristic species. They are interpreted biostratigraphically using Martini's (1971) standard zonal scheme. All palaeontological determination of nannofossils, as well as biostratigraphic interpretation and conclusions are implemented by K. Stoykova.

\section{Geological setting}

The studied area is a part of the Pindos foreland. It belongs to the external Ionian zone of the Hellenides, and comprises the three Diapondia islands - Othonoi, Mathraki and Erikoussa (NW Greece). To the north it is bounded by the Borsh-Khardhiqit strike slip fault, to the south by the Corfu margin, to the east by the Middle Ionian thrust, and to the west by the Ionian thrust. Therefore the studied area is situated backward of the Ionian thrust and corresponds to a piggy back basin, where sedimentation is continuous (Fig. 1).

The sedimentation in this basin (Fig. 2) is characterized by fine-grained sediments with channelized sandstone intercalations. These deposits are interpreted as inner fan deposits (Makrodimitras and Zelilidis, 2009) accumulated in the external Pindos foreland, resulted from the segmentation of the Pindos foreland basin due to internal thrusting during Oligocene, which migrated in a westward direction (Underhill 1985; 1989, Clews 1989, Alexander et al. 1990, Avramidis 1999).

According to Makrodimitras and Zelilidis (2009) the sedimentation of submarine fans was continuous in the studied part of the foreland basin, before the Ionian thrust activity. Due to the Ionian thrust activity the western part of the area was uplifted and the deformation of turbiditic sequence was started. Especially, in Mathraki Island the whole sedimentary sequence has strongly deformed, whereas in Erikousa Island two slump horizons were produced, the lower one up to $5 \mathrm{~m}$ thick and $70 \mathrm{~m}$ long, and the upper one, up to $10 \mathrm{~m}$ thick and more than $100 \mathrm{~m}$ long. Both slump horizons show an eastward thinning trend, and consist of thick sandstone clasts. In Mathraki Island the strongly deformed sedimentary sequence is in contact with Triassic evaporates, which came up due to Ionian thrust activity, whereas in Othoni Island Mesozoic limestones outcropped. 

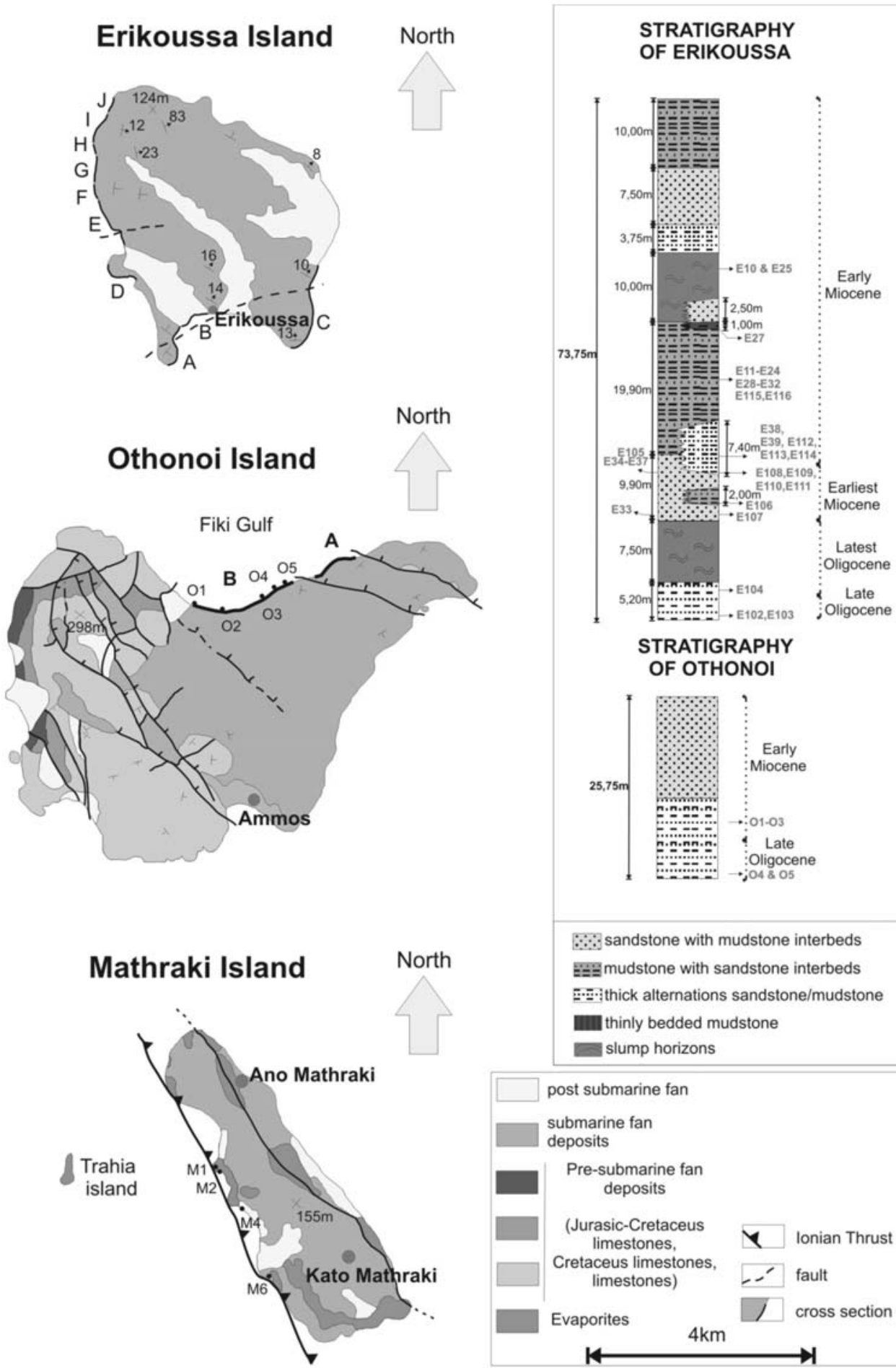

$\therefore$ sandstone with mudstone interbeds I:Izmudstone with sandstone interbeds F-:-ithick alternations sandstone/mudstone

IIIII thinly bedded mudstone slump horizons

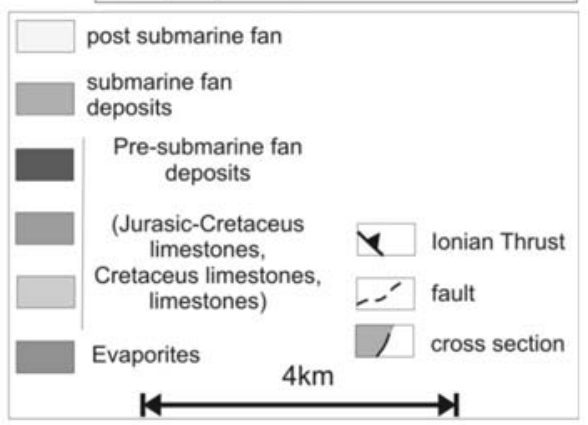

Fig. 2: Geological maps of Erikoussa, Othonoi and Mathraki Islands. 

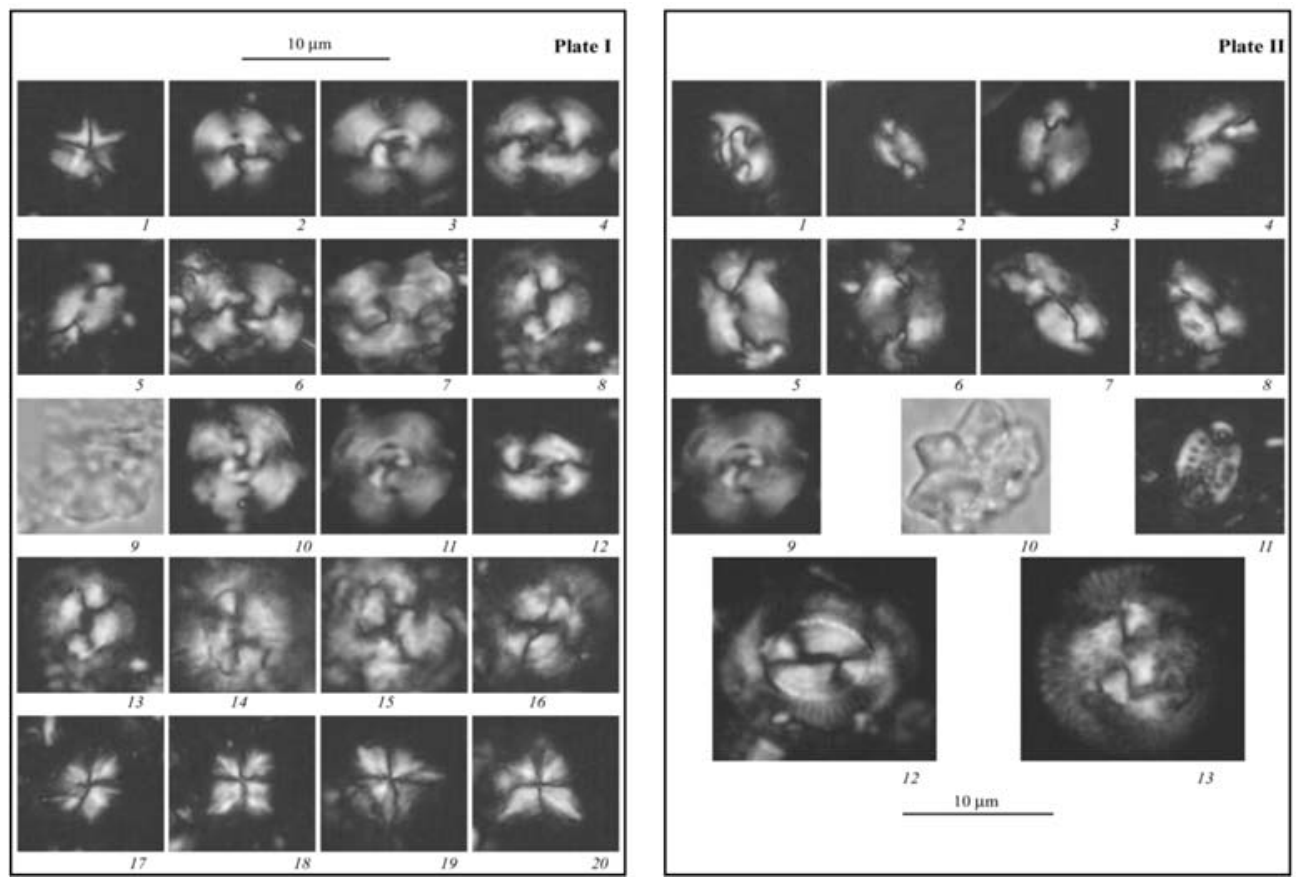

Plates I \&II: nannofossil species from Othonoi Island

Plate I: 1. Micrantholithus sp. indet., sample O5, c.p. 2-3. Cyclicargolithus floridanus 2 - sample O5, c.p. 3 sample O3, c.p. 4. Reticulofenestra retiformis, sample O5, c.p. 5. Helicosphaera sp. aff. obliqua, sample O5, c.p. 6. Cyclicargolithus floridanus, sample O4, c.p. 7. Dictyococcites bisectus, sample O4, c.p. 8. Coccolithus pelagicus, sample O4, c.p. 9. Discoaster adamanteus, sample O4, t.1. 10-11. Cyclicargolithus abisectus, 10 sample O4, 11 - sample 106, c.p. 12. Reticulofenestra retiformis, sample O2, c.p. 13. Coccolithus pelagicus, sample O4, c.p. 14-16. Coccolithus eopelagicus, sample O4, c.p. 17-18. Sphenolithus disbelemnos, sample O3; 17- c.p with angle $45^{\circ} ; 18$ - c.p with angle $90^{\circ}$. 19-20. Sphenolithus conicus, sample 106, 19 - c.p. with angle $45^{\circ} ; 20$ - c.p with angle $90^{\circ}$ (Abbreviations: c.p. $=$ cross-polarised light; t.l. $=$ transmitted light.

Plate II: 1. Helicosphaera sp. aff. elongata, sample O3, c.p. 2. Helicosphaera scissura, sample O3, c. p. 3-4. Helicosphaera compacta, sample O3, c.p. 5-6. Helicosphaera perch-nielseniae, sample 03, c.p.7-8. Helicosphaera elongata, sample O2, c.p. 9. Cyclicargolithus abisectus, sample O3, c.p. 10. Discoaster adamanteus, sample O4, t.1. 11. Pontosphaera multipora, sample O3, c.p. 13-14. Coccolithus miopelagicus sample O3, c.p.

Paleocurrent analysis shows both N-S and an E-W trend, indicating the Ionian thrust activity influence on depositional conditions.

\section{Methodology}

The determination of the sediment ages is based on the study of calcareous nannofossils, originating from 63 samples, distributed across the geological sections along the coastline of the three islands. All samples were processed and smear-slides were prepared using Canada Balsam as a fixative. Each slide was observed under cross-polarized and normal light using a Zeiss Axioskop 40 Pol microscope at 1250x magnification. 

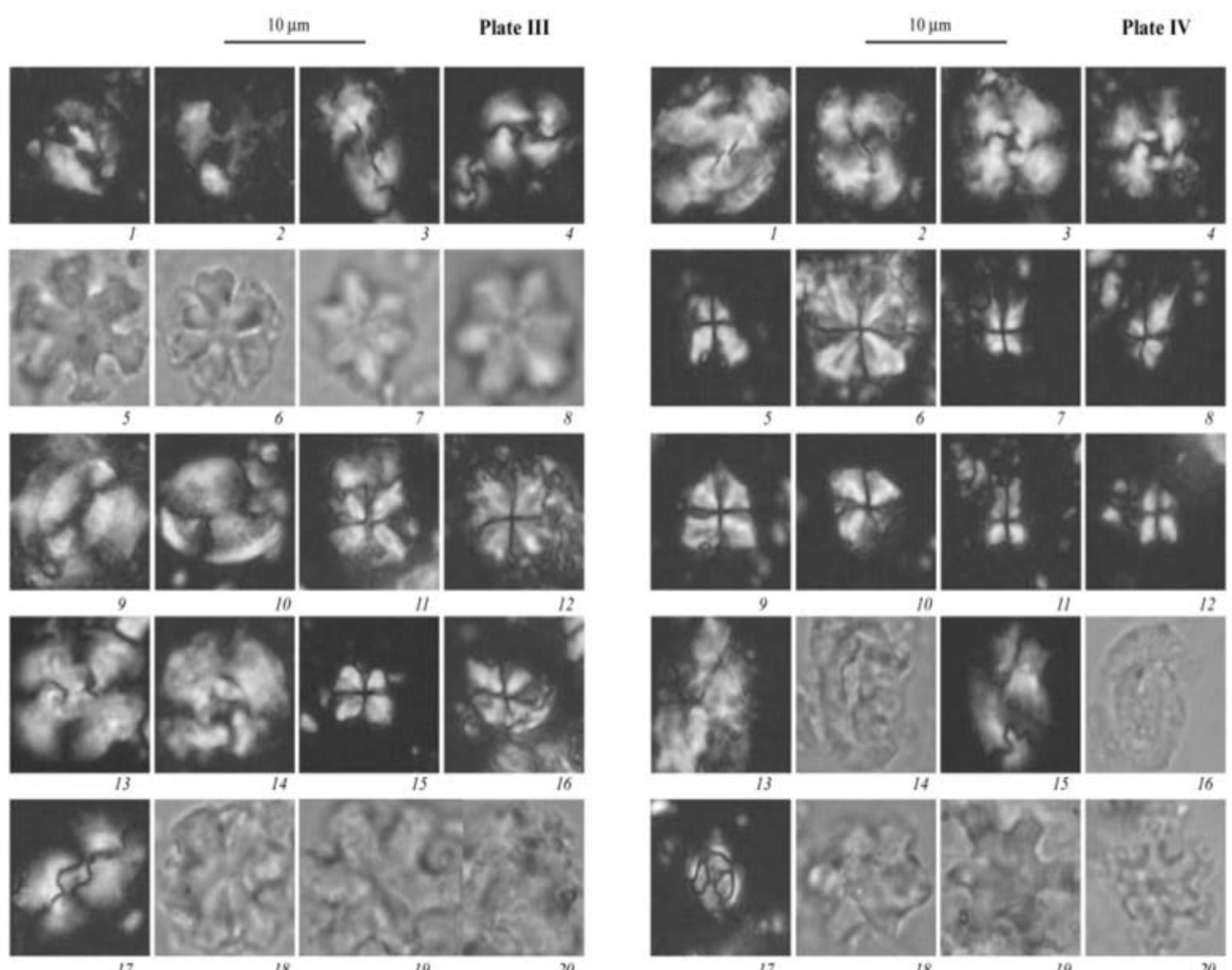

13
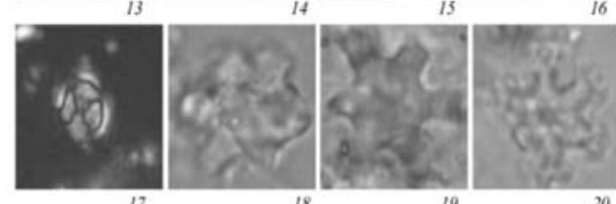

Plates III \& IV: nannofossil species from Erikoussa Island

Plate III: 1-2. Helicosphaera mediterranea, sample E1, outcrop A, c.p. 3. Helicosphaera kamptneri, sample E1, outcrop A, c.p. 4. Reticulofenestra retiformis + Dictyococcites, sample E1, outcrop A, c.p. 5. Discoaster deflandrei, sample E1, outcrop A, t.1. 6. Discoaster cf. calculosus, sample E1, outcrop A, t.1. 7-8. Discoaster adamanteus, 7 - sample E2, 8 - samle 3, t.1.9. Coccolithus eopelagicus, outcrop A, c.p. 10. Pontosphaera multipora, sample E1, outcrop A, c.p. 11-12. Sphenolithus moriformis, sample E4, outcrop A, c.p. 13. Dictyococcites bisectus, sample E2, reworked species in Lower Miocene, c.p. 14. Cyclicargolithus abisectus, sample E1, outcrop A, c.p. 15-16. Sphenolithus conicus, 15 - sample E2, 16 - sample E4, outcrop A, c.p. 17. Helicosphaera euphratis, sample E7, outcrop A, c.p. 18-20. Discoaster calculosus, 18- sample E4, 19, 20 - sample E3, outcrop A, t.1.

Plate IV (outcrop C, Fig. 2): 1-2. Dictyococcites bisectus, sample E103, outcrop C, c.p. 3-4. Cyclicargolithus abisectus, sample E103, outcrop C, c.p. 5. Sphenolithus aff. calyculus sample E105, outcrop C, c.p. 6. Sphenolithus moriformis, sample E106, outcrop C, c.p. 7-8. Sphenolithus dissimilis, sample E106, outcrop C, c.p., 8angle 45 . 9-10. Sphenolithus conicus, sample E106, E107, outcrop C, c.p. 11. Sphenolithus calyculus, sample E107, outcrop C, c.p. 12. Sphenolithus disbelemnos, sample E113, outcrop C, c.p. 13-14. Helicosphaera kamptneri, sample E106, c.p. and t.1. 15-16. Helicosphaera carteri, sample E113, outcrop C, c.p. and t.1. 17. Clausicoccus fenestratus, sample E108, outcrop C, c.p. 18. Discoaster adamanteus, sample E106, outcrop C, t.1. 19. Discoaster cf. drugii, sample E109, outcrop C, t.1. 20. Discoaster aff. deflandrei, sample E113*, outcrop C, t.l.

Biostratigraphic interpretations are based on the first and last occurrence of stratigraphically important nannofossil taxa. In order to achieve accurate age, standard biozonation of Martini (1971) is employed. 


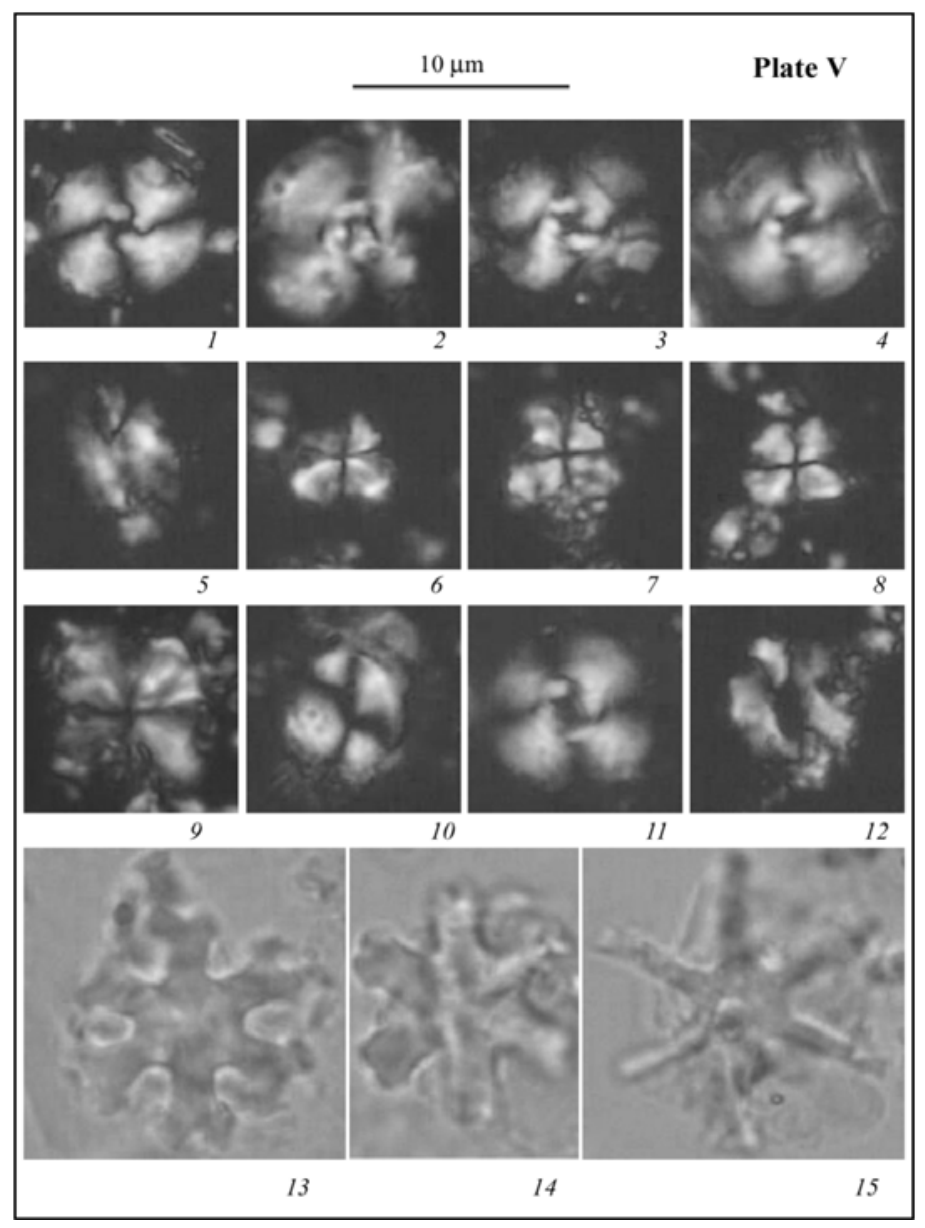

Plate V: nannofossil species from Erikoussa island

Plate V (Outcrop C, Fig. 2): 1-2. Cyclicargolithus floridanus/ abisectus, sample E107, outcrop C, c.p. 3-4. Cyclicargolithus abisectus, sample E108, outcrop C, c.p. 5. Helicosphaera kamptneri, sample E109, outcrop C, c.p. 6-8. Sphenolithus heteromorphus, sample E110, outcrop C, c.p. 9. Sphenolithus moriformis, sample E111, outcrop C, c.p. 10. Coccolithus pelagicus, sample E116, outcrop C, c.p. 11. Cyclicargolithus floridanus/ abisectus, sample E113, outcrop C, c.p. 12. Helicosphaera ampliaperta, sample E116, outcrop C, c.p. 13. Discoaster deflandrei, sample E109, outcrop C, t.1. 14. Discoaster musicus, sample E109, outcrop C, t.1. 15. Discoaster petaliformis, sample E113, outcrop C, t.l.

\section{Results}

\subsection{Erikoussa stratigraphy}

In Erikoussa 41 samples have been studied in order to form the island stratigraphy. These samples have been chosen according to their position in the stratigraphic column to cover the entire succession. The results of the biostratigraphical analysis are shown in the table 1, whereas the selected samples and studied sections are shown in table 2. 
Table 1. Erikoussa samples age determination.

\begin{tabular}{|c|c|c|}
\hline Sample & $\begin{array}{c}\text { Age (NP and NN } \\
\text { zones of Martini, } \\
\text { 1971) }\end{array}$ & Nannofossil species in the association in situ \\
\hline $\begin{array}{l}\text { E11-E24, } \\
\text { E28-32, } \\
\text { E116 }\end{array}$ & $\begin{array}{l}\text { Early Miocene, } \\
\text { NN1-NN4 }\end{array}$ & $\begin{array}{l}\text { Cyclicargolithus abisectus, Cy. floridanus, Sphenolithus moriformis, S. compactus, S. conicus, } \\
\text { Bicolumnus ovatus, Coccolithus pelagicus, C. miopelagicus, Helicosphaera granulata, H. } \\
\text { kamptneri, Discoaster sp, Discoaster deflandrei, D. druggi, D. calculosus, Sphenolithus dis- } \\
\text { similis, Reticulofenestra daviesi, Pontosphaera multipora, S. calyculus, Pyrocyclus orangensis, } \\
\text { Discoaster adamanteus, Helicosphaera intermedia, Reticulofenestra minuta, small-sized Retic- } \\
\text { ulofenestra, Helicosphaera californiana, Braarudosphaerabigelowii, Micrantholithus spp, He- } \\
\text { licosphaera paleocarteri, Sphenolithus disbelemnos (NN2-3), Sphenolithus belemnos, } \\
\text { Coccolithus eopelagicus, Sphenolithus heteromorphus, H. elongata, H. carteri, H. Carteri } \\
\text { hyalina, H. euphratis, H. ampliaperta, Calcidiscus leptoporus. }\end{array}$ \\
\hline E102-E104 & $\begin{array}{l}\text { Late Oligocene to } \\
\text { Earliest Miocene, } \\
\text { NP24-NN1 }\end{array}$ & $\begin{array}{l}\text { Coccolithus pelagicus, C. eopelagicus, Cyclicargolithus floridanus, Cy. abisectus, Sphenolithus } \\
\text { moriformis, Dictyococcites bisectus, D. scrippsae, Helicosphaera compacta, H. intermedia, H. } \\
\text { euphratis, Pontosphaera multipora, Reticulofenestra retiformis, Helicosphaera kamptneri, Dis- } \\
\text { coaster deflandrei, Discoaster cf. calculosus, Transversopontis sp. indet. }\end{array}$ \\
\hline E106 & $\begin{array}{l}\text { Early Miocene, } \\
\text { NN1 }\end{array}$ & $\begin{array}{l}\text { Discoaster adamanteus, D. deflandrei, Cyclicargolithus abisectus, Sphenolithus moriformis, } S \text {. } \\
\text { calyculus, S. conicus, S. pacificus, S. dissimilis, Dictyococcites bisectus, Reticulofenestra reti- } \\
\text { formis, Coccolithus pelagicus, Helicosphaera kamptneri, Pontosphaera multipora. }\end{array}$ \\
\hline $\begin{array}{c}\text { E33-37, } \\
\text { E105,E107 }\end{array}$ & \begin{tabular}{l|} 
Latest Oligocene to \\
Early Miocene, \\
NP25 to NN3
\end{tabular} & $\begin{array}{l}\text { Coccolithus pelagicus, C. miopelagicus, Cyclicargolithus abisectus, Cy. floridanus, Heli- } \\
\text { cosphaera compacta, H. euphratis, Sphenolithus compactus, S. moriformis, S. conicus, S. caly- } \\
\text { culus, Pontosphaera multipora, Discoaster calculosus, Dictyococcites bisectus, D. scrippsae, C. } \\
\text { eopelagicus, R. daviesi,Pyrocyclus orangensis,Helicosphaera kamptneri, Reticulofenestra min- } \\
\text { uta, small-sized Reticulofenestra, S. compactus, Discoaster druggii, Helicosphaera paleocarteri. }\end{array}$ \\
\hline \begin{tabular}{|l} 
E38,E39, \\
E108-E114
\end{tabular} & $\begin{array}{l}\text { Early Miocene, } \\
\text { NN1-NN3 }\end{array}$ & $\begin{array}{l}\text { Sphenolithus disbelemnos, S. dissimilis, S. moriformis, S.conicus, S. compactus, Cyclicargolithus } \\
\text { abisectus, Cy.floridanus, Helicosphaera kamptneri, H.intermedia, Discoaster deflandrei, Coc- } \\
\text { colithus pelagicus,Coccolithus miopelagicus, Reticulofenestra minuta, Pyrocyclus orangen- } \\
\text { sis,H. euphratis,Clausicoccus fenestratus, Pontosphaera multipora, C. eopelagicus, } \\
\text { Pontosphaera multipora, Discoaster deflandrei, small-sized Reticulofenestra, Dictyococcites } \\
\text { scrippsae, D. calculosus, Discoaster druggii. }\end{array}$ \\
\hline $\begin{array}{c}\text { E115, } \\
\text { E30-E32 }\end{array}$ & $\begin{array}{l}\text { Early Miocene, } \\
\text { NN1-NN5 }\end{array}$ & $\begin{array}{l}\text { Small-sized Reticulofenestra, Sphenolithus disbelemnos, Coccolithus pelagicus, Cyclicargolithus } \\
\text { abisectus, Cy. floridanus, Helicosphaera kamptneri, H.carteri hyalina, Reticulofenestra min- } \\
\text { uta,Discoaster adamanteus, Coccolithus miopelagicus, C.eopelagicus, R. daviesi, S. dissimilis, } \\
\text { Discoaster deflandrei, Calcidiscus leptoporus, Pontosphaera multipora. }\end{array}$ \\
\hline E27 & $\begin{array}{l}\text { Early Miocene, } \\
\text { NN3 }\end{array}$ & $\begin{array}{l}\text { Sphenolithus belemnos, S. conicus, S. moriformis, S. compactus, Cyclicargolithus abisectus, } \\
\text { Calcidiscus leptoporus, Coccolithus pelagicus, Helicosphaera intermedia, H. euphratis, Dis- } \\
\text { coaster calculosus, D. deflandrei, Reticulofenestra daviesi, C. eopelagicus, C. miopelagicus. }\end{array}$ \\
\hline E25, E10 & $\begin{array}{l}\text { Early Miocene, } \\
\text { NN1-NN2 }\end{array}$ & $\begin{array}{l}\text { Discoaster calculosus, D. deflandrei, Sphenolithus moriformis, S. dissimilis, S. compactus, Coc- } \\
\text { colithus pelagicus, C. miopelagicus, Cy. floridanus, Cy. abisectus, Helicosphaera kamptneri, } \\
\text { H. euphratis, Reticulofenestra minuta, Dictyococcites scrippsae. }\end{array}$ \\
\hline
\end{tabular}


Table 2. Erikoussa outcrops and samples.

\begin{tabular}{|l|l|}
\hline Erikoussa outcrops & Samples \\
\hline C & $102,103,104,105,107,108,109,110,111,113,114,115$ \\
\hline D & $10,11,12,13,14,15,16,17,18,19,20,21,22,23,24$ \\
\hline E & 25,27 \\
\hline F & $30,31,32$ \\
\hline G/H/I/J & $33,34,35,36,37,38,37$ \\
\hline
\end{tabular}

\subsection{Othonoi stratigraphy}

In Othonoi outcrop B, 4 samples have been studied. The sample $\mathrm{O} 1$ is from the contact of submarine fan deposits with the Pre-submarine fan deposits to the west (Cretaceous limestones). The results of the analysis are shown in the table 3:

Table 3. Othonoi outcrop B age determination.

\begin{tabular}{|c|c|c|c|}
\hline Sample & $\begin{array}{l}\text { Age (NP and NN } \\
\text { zones of Martini, } \\
\text { 1971) }\end{array}$ & Nannofossil species in the association in situ & $\begin{array}{l}\text { Depositional environ- } \\
\text { ment/ reworking/ } \\
\text { remarks }\end{array}$ \\
\hline 01 & $\begin{array}{l}\text { Early Miocene, } \\
\text { NN2-3 }\end{array}$ & $\begin{array}{l}\text { Discoaster druggii, D. calculosus, D. deflandrei, Cyclicar- } \\
\text { golithus floridanus, Cy. Abisectus, Sphenolithus dissimilis, S. } \\
\text { moriformis, Coccolithus pelagicus, C. miopelagicus. }\end{array}$ & $\begin{array}{l}\text { Reworking of Upper } \\
\text { Cretaceous, Paleocene } \\
\text { and Oligocene species. }\end{array}$ \\
\hline $\mathrm{O} 2$ & $\begin{array}{l}\text { Early Miocene, } \\
\text { NN1-3 }\end{array}$ & $\begin{array}{l}\text { Coccolithus pelagicus, C. eopelagicus, Cyclicargolithus flori- } \\
\text { danus, Cy. abisectus, Dictyococcites scrippsae, Heli- } \\
\text { cosphaera elongata, H. perch-nielseniae, H. scissura, } \\
\text { Sphenolithus moriformis, Discoaster adamanteus. }\end{array}$ & $\begin{array}{l}\text { Rich and diverse nan- } \\
\text { nofloral association, no } \\
\text { reworking. }\end{array}$ \\
\hline $\mathrm{O} 3$ & $\begin{array}{l}\text { Early Miocene, } \\
\text { NN1-2 }\end{array}$ & $\begin{array}{l}\text { Sphenolithus conicus, S. disbelemnos, Sphenolithus mori- } \\
\text { formis, Coccolithus pelagicus, Cyclicargolithus floridanus, } \\
\text { Cy. abisectus, Helicosphaera compacta, H. scissura, H. sp. } \\
\text { aff. elongata, H.perch-nielseniae, Dictyococcites scrippsae, } \\
\text { Discoaster deflandrei, Pontosphaera multipora, Reticulofen- } \\
\text { estra retiformis. }\end{array}$ & $\begin{array}{l}\text { Recovery of the abun- } \\
\text { dant and diverse nan- } \\
\text { nofloral association, } \\
\text { with very good to excel- } \\
\text { lent preservation. It is } \\
\text { indicative of favourable } \\
\text { conditions for blooming } \\
\text { of the nannoflora. }\end{array}$ \\
\hline $\mathrm{O} 4$ & $\begin{array}{l}\text { Latest Oligocene } \\
\text { to Early Miocene, } \\
\text { NP25-NN1 }\end{array}$ & $\begin{array}{l}\text { Coccolithus pelagicus, C. eopelagicus, Cyclicargolithus flori- } \\
\text { danus, Cy. abisectus, Dictyococcites scrippsae, D. bisectus, } \\
\text { Sphenolithus moriformis, Discoaster adamanteus. }\end{array}$ & $\begin{array}{l}\text { Impoverishment of the } \\
\text { association - poor } \\
\text { preservation with signs } \\
\text { of overgrowth and re- } \\
\text { crystalization. }\end{array}$ \\
\hline 05 & $\begin{array}{l}\text { Latest Oligocene } \\
\text { to Early Miocene, } \\
\text { NP25-NN1 }\end{array}$ & $\begin{array}{l}\text { Coccolithus pelagicus, C. eopelagicus, Cyclicargolithus } \\
\text { floridanus, Cy. abisectus, Dictyococcites scrippsae, Retic- } \\
\text { ulofenestra retiformis, } \text { R. Hillae, Sphenolithus moriformis, } \\
\text { Helicosphaera intermedia, Helicosphaera sp. aff.obliqua, } \\
\text { Discoaster adamanteus, D.cf.calculosus, D. deflandrei, Mi- } \\
\text { crantholithus sp. indet. }\end{array}$ & $\begin{array}{l}\text { Rich and diverse nan- } \\
\text { nofloral association, no } \\
\text { reworking. }\end{array}$ \\
\hline
\end{tabular}




\subsection{Mathraki results}

In Mathraki the thrust activity has destroyed every old turbiditic sequences. Nevertheless, we choose 3 samples from the west coastline destroyed sequences, and one sample (M1) from the contact of submarine fan deposits with the thrust. The results of the analysis are shown in the table 4:

Table 4. Mathraki samples age determination.

\begin{tabular}{|c|c|c|c|}
\hline Sample & $\begin{array}{l}\text { Age (NP and NN } \\
\text { zones of Martini, } \\
\text { 1971) }\end{array}$ & Nannofossil species in the association in situ & $\begin{array}{l}\text { Depositional environ- } \\
\text { ment/ reworking/ } \\
\text { remarks }\end{array}$ \\
\hline M1 & $\begin{array}{l}\text { Late Miocene, } \\
\text { NN8-9 }\end{array}$ & $\begin{array}{l}\text { Discoaster exilis, D. bollii, D. deflandrei, D. druggii, D.cf. } \\
\text { kugleri, Calcidiscus macintyrei, Calcidiscus tropicus, Coc- } \\
\text { colithus pelagicus, Cyclicargolithus floridanus, Cy. abisectus, } \\
\text { Pontosphaera spp., Dictyococcites scrippsae, Sphenolithus } \\
\text { moriformis, Helicosphaera kamptneri. }\end{array}$ & $\begin{array}{l}\text { Rich and diverse nan- } \\
\text { nofloral association, } \\
\text { Minor reworking: D. bi- } \\
\text { sectus (Oligocene), Uni- } \\
\text { planarius gothicus } \\
\text { (Upper Cretaceous). }\end{array}$ \\
\hline M2 & $\begin{array}{l}\text { Latest Oligocene } \\
\text { to Early Miocene, } \\
\text { NP25-NN1 }\end{array}$ & $\begin{array}{l}\text { Coccolithus pelagicus, Cyclicargolithus floridanus, Cy. abi- } \\
\text { sectus, Dictyococcites scrippsae, Sphenolithus moriformis, } \\
\text { Discoaster deflandrei. }\end{array}$ & $\begin{array}{l}\text { Impoverishment of the } \\
\text { association - poor } \\
\text { preservation. Large } \\
\text { amount of Si-skeletons } \\
\text { of microplankton: a lot } \\
\text { of spicules of Si- } \\
\text { sponges, diatoms, radio- } \\
\text { larians are observed. }\end{array}$ \\
\hline M4 & $\begin{array}{l}\text { Early Miocene, } \\
\text { NN1-2 }\end{array}$ & $\begin{array}{l}\text { Discoaster calculosus, D. deflandrei, Sphenolithus com- } \\
\text { pactus, Sphenolithus moriformis, Coccolithus pelagicus, Coc- } \\
\text { colithus miopelagicus, Cyclicargolithus floridanus, Cy. } \\
\text { abisectus, Helicosphaera kamptneri, H. intermedia, H.eu- } \\
\text { phratis, Dictyococcites scrippsae, Pontosphaera multipora, } \\
\text { Reticulofenestra retiformis. }\end{array}$ & $\begin{array}{l}\text { Diverse nannofloral as- } \\
\text { sociation; again abun- } \\
\text { dant Si- skeletons of } \\
\text { microplankton ob- } \\
\text { served. }\end{array}$ \\
\hline M6 & $\begin{array}{l}\text { Early Miocene, } \\
\text { NN1-2 }\end{array}$ & $\begin{array}{l}\text { Helicosphaera kamptneri, Coccolithus pelagicus, } C \text {. } \\
\text { miopelagicus, Discoaster calculosus, D. deflandrei, Cycli- } \\
\text { cargolithus floridanus, Cy. abisectus, Braarudosphaera } \\
\text { bigelowii, Pontosphaera multipora, Sphenolithus moriformis, } \\
\text { S. compactus. }\end{array}$ & $\begin{array}{l}\text { Abundant Si- skeletons } \\
\text { of microplankton. }\end{array}$ \\
\hline
\end{tabular}

\section{Conclusions}

Considering the age determination results of the studied sections we can conclude that:

1. The sedimentation in the studied area occurred during the Late Oligocene to Early Miocene time.

2. The age of the sediments in studied area (external part of Pindos foreland), is younger than the 
other parts situated eastwards, either in the middle or in the internal parts of the Pindos foreland, showing the internal thrusting activity influence on the depositional conditions.

3. The presence of slump horizons in Erikoussa island (Late Oligocene-Early Miocene) and the highly deformed deposits in Mathraki island, overthrusting Triassic evaporites, indicate that Ionian thrust activity started at the end of Oligocene and caused deformation and slumping of the turbiditic sequences.

4. Taking into account that Ionian thrust activity in Zakynthos Island took place during early Pliocene, the different time activities of the different parts of the Ionian thrust could be indicated. These parts are bounded by strike-slip faults and the oldest activity is manifested northwards whereas the younger is shifted southwards.

5. The studied area, with the three islands, changed from a foreland basin that formed in response to the middle Ionian thrust activity, to a piggy back basin, as it is situated on the hangingwall of the active Ionian thrust, after Late Miocene, and when tectonic activity migrated westwards. This piggy-back basin remains unchanged in now days, whereas the new foreland basin, the Ionian foreland, is situated in the footwall of the Ionian thrust.

\section{References}

Alexander J., Nichols G.J., Leigh S., 1990. The origins of marine conglomerates in the Pindos foreland basin, Greece. Sedimentary Geology, 66, 243-254.

Aubouin J., 1959. Contribution à l'étude géologique de la Grèce Septentrionale. Les confines de l'Epire et de la Thessalie, Ann. Geol. des Pays Helen. 10, 1-525.

Avramidis P., 1999. Depositional environments and hydrocarbon potential of Tertiary formations in Klematia-Paramythia basin, Epirus, PhD thesis, University of Patras.

Avramidis, P., Zelilidis, A. 2001: The nature of deep-marine sedimentation and palaeocurrent trends as an evidence of Pindos foreland basin fill conditions. Episodes, 24, No4, 252-256.

Avramidis, P., Zelilidis, A., Vakalas, I. \& Kontopoulos, N. 2002: "Interaction between tectonic activity and eustatic sea-level changes in the Pindos and Mesohellenic Basins, NW Greece: basin evolution and hydrocarbon potential. -Journal of Petroleum Geology, 25 (1), 53-82.

Clews J., 1989. Structural controls on basin evolution: Neogene to Quaternary of the Ionian zone of western Greece. J. Soc. London, 146, 447-457.

IGSR\&IFP, 1966. Etude geologique de l'Epire. Paris: Technip, 306pp.

Makrodimitras G., Zelilidis A., 2009. New aspects for the northwestern part of Pindos foreland evolution, Diapondia islands, Greece. 27th IAS meeting of Sedimentology 2009, Alghero - Italy, 260.

Martini E., 1971. Standard Tertiary and Quaternary calcareous nannoplankton zonation. Proceedings of the II Planktonic Conference, Roma, 1970, 2, 739-785.

Vakalas, J., Ananiadis, G., Mpourlokas, J., Poulimenos, D., Getsos, K., Pantopoulos, G., Avramidis, P., Zelilidis, A. \& Kontopoulos, N. 2001: Palaeocurrent directions as an indicator of Pindos foreland evolution (central and southern part), Western Greece. - Bulletin of the Geological Society of Greece, Vol. XXXIV/2, 785-791.

Underhill J. R., 1989. Late Cenozoic deformation of the Hellenide foreland, western Greece. Geol. Soc. of Amer. Bull., 101, 613-634. 